\section{ADESÃO DA GESTANTE AO EXERCÍCIO FÍSICO PARA A PREVENÇÃO E/OU CONTROLE DO RISCO DA SÍNDROME HIPERTENSIVA}

\author{
Adhesion of the pregnant to the physical exercise for the \\ prevention and / or control of the risk of hypertensive syndrome
}

\section{RESUMO}

Objetivo: Analisar as mudanças comportamentais em gestantes na adesão ao exercício físico com vista à prevenção e/ou controle do risco da síndrome hipertensiva na gravidez (SHG) com a utilização de uma tecnologia educativa em saúde (TES) embasada na Teoria Estágio de Mudança. Métodos: Pesquisa participante realizada em uma Unidade de Atenção Primária em Fortaleza-Ceará-Brasil com um grupo de dez gestantes no período de agosto a outubro de 2015. A TES foi elaborada com base no referencial de Prochaska; Norcross; Diclemente e nos pressupostos da educação em saúde. Os dados foram coletados por meio de entrevistas e diário de campo, e organizados nas categorias: caracterização sociodemográfica, sanitária e obstétrica das mulheres; resgate das experiências prévias com o exercício físico; mudanças ocorridas com a aplicação da TES com vista à adesão ao exercício físico; avaliação da prática do exercício físico; re-significação da TES para a adesão do exercício físico na prevenção e/ou controle do risco da SHG. Resultados: As gestantes nasceram em Fortaleza-Ceará-Brasil e as demais em outros municípios do Estado do Ceará. Seis eram adolescentes e quatro adultas. As gestantes informaram fatores de risco para SHG - baixo nível socioeconômico, duas eram obesas, cinco consumiam alimentos com alto teor de sal, sete eram sedentárias, duas afirmaram história familiar da SHG, e uma era portadora de hipertensão arterial sistêmica. As mulheres encontravam-se no curso gestacional entre 16 semanas e 28 semanas. O saber das gestantes acerca do EF era fragmentado. Com a aplicação da TES, em quase todas as gestantes percebemos a adesão ao EF, assim como a construção do saber sobre os benefícios para a sua saúde, tornando-as agente multiplicadoras. Conclusão: Logo, a aplicação da TES conduziu as gestantes ao "estágio de manutenção", significando que elas aderiram ao EF, como conduta promotora de saúde. No entanto, ainda é necessário incentivo para mantê-las neste estágio, elevando-as à terminação, mediante a incorporação desta conduta no seu estilo de vida.

Descritores: Adesão; Exercício; Hipertensão Induzida pela Gravidez.

\section{ABSTRACT}

Objective: To analyze behavioral changes in pregnant women in adherence to physical exercise in order to prevent and/or control the risk of hypertensive syndrome in pregnancy (HSP) with the use of a health education technology (HET) based on the Stage Theory of Change. Methods: Participant research carried out in a Primary Care Unit in FortalezaCeará-Brazil with a group of ten pregnant women from August to October 2015. The HET was elaborated based on the Prochaska reference; Norcross; Diclemente and in the assumptions of health education. The data were collected through interviews and field diaries, and organized into the categories: sociodemographic, sanitary and obstetric characterization of women; Recovery of previous experiences with physical exercise; Changes that occurred with the application of HET in view of adherence to physical exercise; Evaluation of physical exercise practice; Re-signification of the HETto the adhesion of the physical exercise in the prevention and / or control of the risk of HSP. Results: The pregnant women were born in Fortaleza-Ceará-Brazil and the others in other municipalities in the State of Ceará. Six were teenagers and four were adults. Pregnant women reported risk factors for HSP-low socioeconomic status, two were obese, five consumed foods high in salt, seven were sedentary, two reported a family history of HSP, and one had systemic arterial hypertension. The women
Artigo Original

Aline Barbosa Teixeira Martins ${ }^{(1)}$

Zélia Maria de Sousa Araújo Santos $^{(1)}$

Geraldo Bezerra da Silva Junior $^{(1)}$

Geraldo Flamarion da Ponte Liberato Filho ${ }^{(1)}$

Maria Vieira de Lima Saintrain ${ }^{(1)}$ José Manuel Peixoto Caldas ${ }^{(2)}$ Italo Rigoberto Cavalcante Andrade $^{(1)}$

Janaina da Silva Feitoza Palácio(1) $^{(1)}$

1) Universidade de Fortaleza - UNIFOR Fortaleza (CE) - Brasil

2) Universidade de Porto - UP - Porto Portugal

Este artigo seguiu as normas e formatação estabelecidas pelo $5^{\circ} \mathrm{CIAIQ}$ - Congresso Ibero-Americano em Investigação Qualitativa. 
were in the gestational period between 16 weeks and 28 weeks. The knowledge of pregnant women about PE was fragmented. With the application of TES, in almost all pregnant women we perceive adherence to $P E$, as well as the construction of knowledge about the benefits to their health, making them multiplying agents. Conclusion: Therefore, the application of HET led the pregnant women to the "maintenance stage", meaning that they adhered to the PE, as a health promoter. However, there is still an incentive to keep them at this stage, raising them to completion by incorporating this behavior into their lifestyle.

Descriptors: Adhesion; Exercise; Hypertension PregnancyInduced.

\section{INTRODUÇÃO}

As síndromes hipertensivas na gravidez (SHG) são as causas primordiais de mortalidade materna (MM) por obstétricas indiretas, ou seja, aquelas resultantes de doenças prévias a gestação ou de agravos ocorridos durante a gravidez, não devidas a causas obstétricas diretas, mas que foram exacerbadas pelos efeitos fisiológicos da gestação $^{(1)}$.

A SHG é definida a partir dos valores absolutos de pressão arterial (PA) sistólica acima de $140 \mathrm{mmHg}$ e/ou PA diastólica acima de $90 \mathrm{mmHg}$, considerando o $5^{\circ}$ ruído de Korotkoff, confirmada por outra medida realizada com intervalo de 4 horas $^{(2)}$.

O Ministério da Saúde divulgou que em 2007, a razão da mortalidade materna (RMM) condiz a 75 mortes/100.000NV, correspondente a 1.572 óbitos ocorridos, em que as SHG são as principais causas ${ }^{(3)}$. No entanto, uma boa parte destas mortes poderiam ser evitadas, se a assistência à gestação, ao parto e ao puerpério fosse $\operatorname{adequada}^{(4-6)}$.

Ainda não há dados precisos acerca da incidência de pré-eclâmpsia (PE), porém, supõe-se que atinja cerca de 4,0\% das gestações. Já no Brasil existem dados de 1,5\% para $\mathrm{PE}$ e $0,6 \%$ para eclampsia (EC), enquanto em áreas desenvolvidas possui incidência de $0,2 \%$ para $\mathrm{EC}$, com índice de $\mathrm{MM}$ de $0,8 \%$, enquanto em áreas subdesenvolvidas apresentam $8,1 \%$ e $22 \%$, respectivamente ${ }^{(2)}$. Além disto, as SHG resultam em mortalidade entre 20,0 e $25,0 \%$ de todas as causas de óbito materno, com tendência a estagnação ${ }^{(7)}$.

O Brasil ainda apresenta taxas de morbimortalidade materna e neonatal (MMN) muito elevadas, sendo considerado um indicador de baixo nível socioeconômico do País. Desse modo, consiste também um desafio para o Sistema de Saúde (SS), em que muito mais do que a redução de índices, busca-se a diminuição de MMN. A maioria das mortes e complicações que aparece no período gestacional, parto e puerpério é de caráter prevenível, todavia para se alcançar esta meta, é necessária a participação direta do $\mathrm{SS}^{(8)}$.

De acordo com dados do Departamento de Informática do Sistema Único de Saúde (DATASUS/MS), no Brasil a SHG é a primeira causa de morte materna especialmente, quando se instalam nas suas formas mais graves. Representa a entidade clínica de maior obituário perinatal, acarretando, ainda, substancial número de neonatos vitimados, e quando sobrevivem aos danos da hipóxia perinatal ${ }^{(9)}$. E conforme dados do Sistema de Informação sobre mortalidade (SIM/ MS), a RMM para 2007 foi de 77,0/100.000 nascidos vivos, apontando a SHG como sendo a maior causa de morte materna no País, responsável por cerca de $20,0 \%$ da taxa de 77,0 mortes materna/100.000 nascidos vivos ${ }^{(10)}$.

Como parâmetro para atenuação das altas taxas de MM, o Governo Federal projetou estratégias para diminuílas com o lançamento do Pacto pela Vida, tendo como propósito e metas a atenuação em 5,0\% a RMM, com a garantia de insumos e medicamentos para tratamento das $\mathrm{SHG}^{(11)}$. A Equipe Saúde da Família (EqSF) possui diversas condutas e uma de maior importância é a assistência às gestantes na consulta de pré-natal $(\mathrm{PN})$, em que essas são auxiliadas com vista à promoção do bem-estar físico e mental, por meio de instruções apropriadas acerca do desenvolvimento gestacional e fetal, e reconhecimento dos fatores que induzam à intercorrência gestacional, em particular a $\mathrm{SHG}^{(12)}$.

$\mathrm{Na}$ década de 80, foi implantado o Programa de Assistência Integral à Saúde da Mulher (PAISM) que não contemplou a gestante, surgindo então, a necessidade de um programa para PN e parto. Dessa forma, em 2000 foi criado o Programa de Humanização no Pré-natal e Nascimento (PHPN $)^{(13,14)}$ com intuito de garantir o avanço do acesso, da cobertura, da qualidade, do acompanhamento PN, e da assistência ao parto e puerpério ${ }^{(15)}$. O objetivo primordial do PHPN é assegurar que a mulher tenha o resgate da dignidade no espaço do processo parturitivo, buscando consolidar a transformação da atenção prestada durante a gestação, o parto e o puerpério, tendo como finalidade incentivar um atendimento obstétrico integral garantindo os direitos de escolha dessa usuária ${ }^{(16,8)}$. As gestantes têm direito ao acesso e devem ser comunicadas de todo e quaisquer serviços de saúde, como: palestras, programas, atividades, orientação quanto à sexualidade, riscos de aborto, fisiologia da reprodução, prevenção de doenças, higiene, dentre essas, ações voltadas para as $\mathrm{SHG}^{(8,17,18)}$.

Diante da necessidade de garantir que as políticas nacionais fossem realmente executadas e viessem a responder às reais necessidades locais da população, o Ministério da Saúde(MS) propôs a adoção do Pacto Nacional pela Redução da MMN. Este pacto visa incluir como 
obrigatoriedade e rotina, uma série de ações estratégicas capazes de reduzi-la. Vários países em desenvolvimento já conseguiram obter excelentes resultados na melhoria de seus indicadores por meio de ações organizadas, amplas, integradas e com cobertura abrangente, utilizando tecnologias simplificadas e economicamente viáveis. A redução da MMN está diretamente relacionada ao acesso das gestantes ao atendimento do PN de qualidade e em tempo oportuno, no nível de complexidade necessário ${ }^{(19)}$.

Considerando a importância da gravidez de alto risco no binômio saúde-doença materno-fetal, é necessária a identificação precoce destas gestações, para que assim seja possível proporcionar uma assistência médica adequada e obter resultados satisfatórios, uma vez que, os óbitos decorrentes das complicações da gravidez são, em sua maioria, preveníveis ${ }^{(20)}$.

As estratégias para prevenção devem ser tomadas para o tratamento da SHG através de políticas públicas de saúde associada às ações da sociedade médica e de comunicação com intuito de estimular o diagnóstico precoce, o tratamento contínuo, o controle da PA por meio da modificação do estilo de vida e/ou uso regular de medicamentos ${ }^{(2)}$.

$\mathrm{O}$ estilo de vida insalubre dessas mulheres vem a contribuir para o agravamento da SHG, visto que elas desconhecem condutas importantes para o controle desta, como a alta ingesta de sal, sedentarismo, tabagismo, alcoolismo, de modo que vem a praticá-las, frequentemente, aumentando, assim, a chance de desenvolver a $\mathrm{SHG}^{(21)}$. Este fato é preocupante e só vem a corroborar com o aumento das taxas de morbimortalidade materna associada à entidade clínica, que continua sendo a principal causa.

Dentre as condutas de prevenção e/ou controle da SHG, a prática regular de exercício físico (EF) pode ser uma estratégia adotada durante a gestação, já que mulheres fisicamente ativas apresentam redução do risco de desenvolver SHG em aproximadamente $35,0 \%{ }^{(22)}$. No entanto, algumas intercorrências no curso da gestação determinam contraindicações para a prática de EF em solo ou aquático pelo fato desta, induzir ao risco de abortamento e complicações clínicas ${ }^{(23)}$.

Entretanto, o EF na gestação ainda é alvo de muitas discussões em todo o mundo, visto ser uma população delicada de lidar. Pesquisas demonstram que o EF é capaz de diminuir os níveis de PA através da redução do stress oxidativo, de transformar a resposta imune e melhorar a disfunção endotelial. Dessa forma, o EF na gestação deve ser acompanhado por um profissional de saúde qualificado, capaz de lidar com esse público, promovendo, assim, a essa gestante uma prática segura e prazerosa.

Antigamente, as recomendações de EF para gestantes variavam de acordo com os contextos socioculturais vividos na época. Dessa forma, existiram períodos em que havia contraindicações para esta prática. A maioria dos profissionais da área de saúde temia que a prática de EF acarretasse prejuízos para o curso do ciclo gravídicopuerperal e aconselhavam, por conta disso, o repouso absoluto para todas as gestantes. A partir do Século XX começaram a serem desenvolvidos os programas de assistência com o objetivo de quebra com o círculo vicioso medo-tensão-dor, passando, assim, a incluir o EF como importante nesse processo ${ }^{(24)}$.

O EF na gestação é recomendado na total ausência de qualquer anormalidade, mediante avaliação médica especializada $^{(25)}$. As contraindicações absolutas são o sangramento uterino de qualquer causa, a placentação baixa, o trabalho de parto pré-termo, o retardo de crescimento intrauterino, os sinais de insuficiência placentária, a rotura prematura de membranas e a incompetência istmo-cervical. Já as contraindicações relativas são hipertensão arterial essencial descontrolada, anemia, doenças tireoidianas, diabetes mellitus descompensado, obesidade mórbida e histórico de sedentarismo extremo. Durante uma gestação normal, quem já praticava EF pode continuar a fazê-lo, adequando a prescrição à gestação.

A mulher, por sua vez, pode se beneficiar de forma física e psicológica, mantendo uma parte de seu condicionamento pré-gestacional e diminuição da percepção da dor durante o trabalho de parto. É necessário avaliar a capacidade física da gestante antes de iniciar qualquer tipo de EF, para então adequar a intensidade e tipo a serem realizados, assim como minimizar os riscos, de modo a favorecer a essa gestante os benefícios dessa prática ${ }^{(25)}$.

Os objetivos da prática do EF em gestantes são a manutenção da aptidão física e da saúde, a diminuição de sintomas gravídicos, o melhor controle ponderal, a diminuição da tensão no parto e uma recuperação no pósparto imediato mais rápido. Outros benefícios do $\mathrm{EF}$ na gestante são o auxílio no retorno venoso, prevenindo o aparecimento de varizes de membros inferiores e a melhora nas condições de irrigação da placenta. Em estudo de caso controle realizado por Rudra et $\mathrm{al}^{(26)}$ foi comparado o nível de EF antes de engravidar e o risco de desenvolver PE, sendo 244 (PE) e 470 (normotensas). Foi observado que as mulheres que praticavam $\mathrm{EF}$ vigoroso pré-gestacional tiveram 78,0\% menos chance de desenvolver PE. Esta associação foi independente do peso pré-gestacional. Já Sorensen et $\mathrm{al}^{(22)}$ em seu estudo com 201 mulheres (383 controles) que realizavam EF e o seu risco em desenvolver $\mathrm{PE}$, observou que aquelas que praticavam algum tipo de $\mathrm{EF}$ desde o começo da gestação reduziram o risco em 35,0\% de ter PE.

Então, mediante a problemática da SHG e os benefícios do EF na prevenção deste agravo, optamos por este estudo 
com o objetivo de analisar as mudanças comportamentais em gestantes na adesão ao exercício físico com vista à prevenção e/ou controle do risco da síndrome hipertensiva na gravidez com a utilização de uma tecnologia educativa em saúde embasada na Teoria Estágio de Mudança.

A Teoria Estágio de Mudanças (TEM) proposta por Prochaska, Norcross e DiClemente ${ }^{(27)}$ é composta pelos estágios de mudança - pré-contemplação, contemplação, ação, manutenção e terminação.

Esta teoria tem como base fundamental, que o indivíduo tome consciência de seus atos, sinta-se responsabilizado por tal problema, assuma a real necessidade da mudança, e então venha a conseguir realizar a transformação em sua vida. Ao ser conscientizado por essa teoria o indivíduo em processo de transformação realmente decide, por si só, que necessita mudar e age em busca desta ação. Normalmente, este não volta atrás após conseguir ultrapassar os estágios de mudança.

\section{MÉTODOS}

Pesquisa-participante realizada em uma Unidade de Atenção Primária em Saúde (UAPS) da Secretaria Executiva Regional VI (SER VI), em Fortaleza-CearáBrasil, no período de agosto a outubro de 2015.

Participaram do estudo dez gestantes, dentre as trinta contactadas inicialmente, mediante à possibilidade de evasão destas mulheres ao longo da aplicação da Tecnologia Educativa em Saúde (TES). Portanto, durante a pesquisa de campo permaneceram vinte desistiram, destas algumas relataram como motivos: mudança de endereço, separação conjugal, complicações na gravidez, e indisponibilidade de tempo.

A TES foi elaborada com base no referencial teóricometodológico de Prochaska, Norcross e Diclemente ${ }^{(27)}$ e nos pressupostos da educação em saúde. A TES consistiu de quinze encontros, que aconteceram duas vezes por semana com duração média de sessenta minutos. Vale ressaltar que o protocolo da caminhada foi realizado em dez encontros. Buscamos este referencial teórico-metodológico, por considerarmos de grande relevância para explicar a adoção de comportamentos que possam auxiliar na prevenção e/ ou controle das doenças e também predizer a aceitação de recomendações sobre cuidados com a saúde.

Realizou-se a pesquisa de campo em três etapas:

Primeira etapa - convidamos as mulheres para participar de uma reunião, na qual foi expomos a natureza e objetivos da pesquisa, a aplicação da TES, registro da anuência no Termo de Consentimento Livre e Esclarecido (TCLE) e o compromisso com a sua efetiva participação. A participação da gestante no programa de EF foi confirmada mediante a liberação médica registrada em documento. A seguir, aprazamos data e horário convenientes para as mulheres, para a realização da entrevista inicial, cujo roteiro conteve os dados sociodemográficos, sanitários e obstétricos; identificação dos fatores de risco para SHG; conhecimento sobre o EF e experiência com esta prática. Esta etapa finalizou com o aprazamento do primeiro encontro para a implementação da TES.

Segunda etapa - implementamos a TES. Durante os encontros realizamos oficinas educativas e a prática do EF precedida e sucedida de alongamentos. As oficinas educativas foram realizadas por meio de dinâmicas de grupo, objetivando motivar e fortalecer os vínculos afetivos no ambiente educativo, favorecendo assim, a construção coletiva do conhecimento sobre o EF, riscos do sedentarismo, e promoção da saúde. Dentre as modalidades do EF, optamos pela caminhada por ser indicada para a gestante, além ser exequível. A caminhada foi adaptada a partir do protocolo da American Heart Association (AHA), considerando as condições físicas e clínicas de cada gestante. Ressaltamos que antes e após trinta minutos da caminhada monitoramos os seguintes parâmetros: PA, frequência cardíaca $(\mathrm{FC})$ e respiratória (FR). No último encontro, aprazamos a segunda entrevista. A caminhada foi sempre precedida e sucedida do alongamento, e finalizávamos com a dinâmica "resgate de aprendizagem".

\section{Descrição da TES:}

Primeiro e segundo encontros - abordamos os "Riscos do sedentarismo para a saúde da gestante" com o objetivo de despertar nas gestantes a importância do EF para a promoção da sua saúde. Inicialmente aplicamos a "dinâmica de apresentação" entre as gestantes e "técnicas de alongamento dinâmico grupal” (atividades lúdicas).

Nos três primeiros encontros, as gestantes encontravamse nos "Estágios de pré-contemplação e contemplação", pois ainda estavam em processo de conhecer os benefícios do EF para então iniciarmos o protocolo da caminhada.

Terceiro encontro - iniciamos o protocolo de caminhada, que teve aumento gradativo na duração a cada semana, e de acordo com a particularidade de cada gestante.

Quarto encontro - exploramos os "Benefícios do EF para prevenção da SHG" com o intuito de sensibilizar para a importância da adesão ao EF.

Quinto encontro - debatemos sobre os "agravos prevalentes na gestação", com ênfase na SHG, e discutimos sobre os cuidados preventivos e/ou de controle do risco da SHG.

Sexto encontro - abordamos as "Percepções sobre as barreiras e os benefícios da mudança", quanto à prevenção dos fatores de risco da SHG, por meio da dinâmica "papel amassado"(28) com o objetivo de possibilitar às participantes 
a superação das dificuldades para adoção de estilo de vida saudável.

Sétimo encontro - exploramos as indicações e contra-indicações da prática do EF, buscando capacitar as gestantes a se tornarem agentes multiplicadoras de mudanças, e a identificar nos familiares e amigos potenciais transformadores.

Oitavo encontro - discutimos sobre a importância da alimentação saudável, utilizando a dinâmica do "repolho" ${ }^{(28)} \mathrm{com}$ a finalidade de reafirmar os benefícios da mudança.

Nono encontro - realizamos uma sessão interativa sobre prevenção e combate à dependência química (drogas lícitas e ilícitas), buscando conhecimento das gestantes sobre o risco destas drogas na gestação.

Décimo encontro - fizemos uma discussão sobre a "Promoção da saúde e bem-estar geral mediados pela prática do EF com o objetivo de conscientizar as gestantes que o EF é capaz de melhorar as condições físicas e mentais, promovendo-lhes qualidade de vida no período gravídico.

Décimo primeiro encontro - discorremos sobre as várias alternativas para o "Combate ao sedentarismo", entre elas a prática regular do $\mathrm{EF}$, com o objetivo de ressaltar sua importância na prevenção da SHG, além de orientar a prática das suas diversas modalidades.

Décimo segundo encontro - realizamos uma oficina intitulada "Gerenciamento do estresse", como uma das estratégias para a adoção de um estilo de vida saudável, com vistas à prevenção da SHG.

Décimo terceiro encontro - abordamos sobre as particularidades de cada gestante, deixando-as falarem sobre o aprendizado adquirido, as dúvidas e questionamentos relativos às temáticas abordadas anteriormente.

Décimo quarto e décimo quinto encontros incentivamos as gestantes a dar continuidade à prática do $\mathrm{EF}$, enfatizando as mudanças necessárias à prevenção dos fatores de risco da SHG.

Na terceira etapa - Aplicamos as duas entrevistas semi-estruturadas, com o objetivo de avaliar as mudanças de comportamento ocorridas na adesão ao EF e o impacto dessas na promoção da saúde. A primeira, após um mês do último encontro, com as questões norteadoras: Comente sobre a sua participação nos encontros com vista a sua adesão à prática regular do exercício físico; e Discorra sobre a repercussão da sua participação nos encontros em sua saúde e bem-estar. E a segunda, dois meses depois, utilizando o mesmo roteiro da anterior.

Durante a pesquisa de campo, utilizamos o diário de campo para o registro de informações relevantes para a complementação da análise dos dados.

As entrevistas foram gravadas, conforme o consentimento prévio das mulheres e complementadas com as informações registradas durante os encontros no diário de campo.

As informações foram organizadas a partir da análise de conteúdo, seguindo os postulados de Bardin ${ }^{(29)}$ conforme as etapas para a análise e interpretação dos dados coletados: pré-análise - efetuamos uma leitura exaustiva das entrevistas e das anotações no diário de campo; exploração do material - após a leitura, identificamos e construímos as categorias analíticas, e posteriormente selecionamos os trechos de depoimentos considerados relevantes; e tratamento dos resultados - a partir dos resultados obtidos, procedermos à interpretação, fundamentando na TEM, nos pressupostos da educação em saúde, e na literatura selecionada.

A pesquisa foi desenvolvida de acordo com a Resolução 466/12 da Comissão Nacional de Ética em Pesquisa $(\mathrm{CONEP})^{(30)}$, que regulamenta a pesquisa com seres humanos. Às participantes foram assegurados o anonimato e o direito de retirar o consentimento no momento que desejassem. A pesquisa foi aprovada pelo Comitê de Ética da Universidade de Fortaleza - UNIFOR, sob o protocolo $\mathrm{n}^{\circ} 112.941$.

\section{RESULTADOS E DISCUSSÃO}

Para o processamento da análise, os resultados foram agrupados nas categorias: caracterização sociodemográfica, sanitária e obstétrica das mulheres; resgate das experiências prévias com o exercício físico; mudanças ocorridas com a aplicação da TES com vista à adesão ao exercício físico; avaliação da prática do exercício físico; re-significação da TES para a adesão do exercício físico na prevenção e/ou controle do risco da SHG.

\section{Caracterização sociodemográfica, sanitária e obstétrica}

As gestantes residiam na área adstrita da UAPS, destas sete nasceram em Fortaleza-Ceará-Brasil e as demais em outros municípios do Estado do Ceará. Quatro gestantes co-habitavam com mãe e irmãos, duas com o companheiro, duas com o conjugue, uma com sogros e companheiro, e uma com pais e irmãos, seis eram adolescentes (14 a 19 anos) e quatro adultas (20 a 27 anos), oito de cor parda, seis praticantes do catolicismo e as demais eram evangélicas, seis frequentavam a escola e quatro dedicavam-se exclusivamente às atividades próprias do lar, duas estavam casadas formalmente, cinco viviam em união estável e três eram solteiras, e seis concluíram o ensino fundamental.

As gestantes informaram fatores de risco para a $\mathrm{SHG}$, pois oito possuíam baixo nível socioeconômico, duas eram obesas, cinco consumiam alimentos com alto teor de sal, sete eram sedentárias, duas afirmaram história familiar da SHG, e uma era portadora de hipertensão arterial sistêmica 
(HAS) ou hipertensão crônica. Quanto ao esquema vacinal; seis o declararam atualizado, uma estava em atraso, e as outras três não haviam tomado nenhuma vacina. Com relação aos vícios; uma gestante era tabagista e consumia bebidas alcoólicas, porém raramente; e outra gestante era usuária de álcool e drogas ilícitas.

As mulheres encontravam-se no curso gestacional entre 16 semanas e 28 semanas, oito experienciavam a primeira gestação; uma informou quatro gestações, um aborto e três partos, e a outra teve quatro gravidezes a termo. Sete gestantes iniciaram o $\mathrm{PN}$ no primeiro trimestre gestacional, e as demais no segundo. $\mathrm{O}$ número de consultas realizadas variou de uma a três, visto que as gestantes se apresentavam com 16 semanas a 28 semanas, ou seja, algumas haviam iniciado o PN tardiamente, e haviam realizado somente uma consulta de PN, já a gestante com mais idade gestacional havia participado de três consultas.

\section{Resgate das experiências prévias com o exercício físico}

O saber das gestantes acerca do EF era fragmentado, mostrando uma concreta falta de conhecimento a respeito dos benefícios do EF, inclusive na gestação. As gestantes acreditavam que durante o período gravídico, a prática do EF era contra-indicada.

[...] eu escutei uma vez que faz bem à saúde, mas nunca tive uma orientação que exercício físico é bom para a mulher gestante. Muito pelo contrário, pensei que fizesse mal e que não pudesse fazer nada. [...] (G2, 23 anos).

De acordo com Segato et $\mathrm{al}^{(31)}$, a prática de EF na gestação, desde que autorizada pelo médico e acompanhada por um profissional qualificado, propicia incontáveis privilégios para a saúde da mulher como: diminuição do edema corporal, melhoria da circulação sanguínea, aumento do equilíbrio muscular, prevenção da incontinência urinária, fortalecimento da musculatura abdominal, ajuda no controle do peso, colabora no processo de recuperação após a gestação, além de prevenir a diabetes gestacional, a SHG e redução do risco de PE.

\section{Mudanças ocorridas com a aplicação da TES com vista à adesão ao exercício físico}

Com a aplicação da TES, quase todas as gestantes atingiram o "Estágio de manutenção". Para Prochaska, Norcross e Di Clemente ${ }^{(27)}$ nesta fase é necessário trabalhar para consolidar os ganhos obtidos durante a fase da ação (quando se iniciou a prática do EF) e outros estágios, e lutar para evitar lapsos e recaídas. A mudança nunca termina com a "ação". Esta etapa é uma continuação extremamente importante e que pode durar apenas seis meses, com também um tempo de vida. Caso não haja um forte compromisso de "manutenção", certamente haverá recaída, que geralmente irão para a "fase de pré-contemplação ou de contemplação", como observamos em duas gestantes, que após ter tido os seus recém-nascidos (RN) não continuaram com o programa de caminhada regular.

As mesmas informaram que sabem da importância do $\mathrm{EF}$, que sentiram os benefícios deste no próprio corpo. No entanto, alegam a falta de tempo para esta prática. Entendemos que após a chegada do RN realmente fica mais difícil de manter no ritmo de EF, devido os cuidados inerentes a este. De fato, elas ainda não estavam realmente engajadas no processo de mudança, pois caso contrário, encontrariam uma forma, assim como as três gestantes que conseguiram atingir o "estágio de terminação".

O "estágio da terminação é o objetivo final de todos os transformadores. Neste estágio, no caso do sedentarismo já não apresenta qualquer tentação ou ameaça, dessa forma, nunca retornará a esse comportamento, e ainda terá toda a confiança que pode lidar sem medo ou recaída ${ }^{(27)}$. Na "estágio de finalização", tudo isto se aplica sem qualquer esforço contínuo de sua parte. O círculo da mudança acabou, e o agente transformado, ganhou a luta. Dessa forma, observamos nessas gestantes que a prática do $\mathrm{EF}$ se tornou um hábito em suas vidas, e que elas realmente o fazem, devido ao bem-estar e sensação de saúde que este proporcionou na vida delas.

Para Nascimento et $\mathrm{al}^{(32)}$ a gravidez é um período ideal para a modificação de comportamentos, com isso a introdução de um estilo de vida ativo para pessoas previamente sedentárias se torna interessante, inclusive pela manutenção de efeitos em longo prazo.

\section{Avaliação da prática do exercício físico}

Para avaliação da prática do $\mathrm{EF}$, comparamos as informações das gestantes na primeira e segunda entrevistas após o término da aplicação da TES. Então, percebemos a adesão dessas mulheres a prática do $\mathrm{EF}$, assim como também o saber delas frente aos benefícios advindos do EF, e as mudanças que sentiram em sua vida.

\section{[...] eu não sabia que o EF era bom para saúde da mulher grávida, vim aprender aqui no curso [...] (G4, 16 anos).}

[...] Eu aprendi que o EF na gestação é muito importante porque na gestação pode dar pressão alta, pré-eclampsia, eclampsia, essas coisas e aí com o EF ajuda a prevenir, né? [...] (G6, 21anos).

Nas falas das gestantes, observamos a adesão delas à prática do EF com vista à manutenção da boa saúde e dos parâmetros de PA dentro da normalidade. Notamos durante os encontros que as gestantes se preocupavam muito com o aumento dos níveis da PA. Algumas por terem familiares hipertensos, outras por serem hipertensas ou terem tido em 
algum momento, aumento na PA. Dessa forma, os níveis de PA dentro da normalidade era algo que elas realmente se preocupavam.

Ferreira et $\mathrm{al}^{(33)}$ em estudo realizado com 60 gestantes a respeito do conhecimento das mesmas sobre a prática de $\mathrm{EF}$ na gestação, desde que liberada pelo médico e orientada por profissional qualificado, encontrou que quinze delas às vezes praticavam alguma modalidade de EF, dezenove disseram que não praticavam, e vinte seis disseram que sim. Segundo com Alves e Forjaz ${ }^{(4)}$ o EF de baixa e média intensidade normalmente não acarreta nenhum tipo de risco para a mãe e o feto, já para mulheres previamente bem condicionadas e atletas é exequível a prática de EF de intensidade mais elevada sem possíveis riscos fetais e maternos. Dessa forma, a prática de EF pelas gestantes vai depender do seu estado gestacional e do acompanhamento de profissionais capacitados e qualificados que sejam capazes de lhe garantir qualidade e segurança na sua realização.

De acordo com HGus, Fuchs e Moreira ${ }^{(34) ;}$ Matsudo e Matsudo $^{(35)}$, mulheres que se exercitam antes e durante a gestação possuem propensão a adquirir menos peso, também dão à luz a RN mais saudáveis e são capazes de suportar com mais leveza às exigências inerentes à gestação. Matsudo e Matsudo $^{(35)}$ afirmam que toda mulher deve acumular pelo menos trinta minutos de EF diário, de modo contínuo ou acumulado, de intensidade moderada ou leve. Evidencia-se que a grávida ao participar de um programa de esporte ou exercício mais intenso deve primeiramente ser avaliada por uma equipe médica multidisciplinar, com exames clínicos e físicos, tais como: sistema cardiovascular, pulmonares, metabólicos e musculoesqueléticos.

Para Batista et $\mathrm{al}^{(36)}$ ainda não existem recomendações padronizadas de EF durante o período gestacional. É recomendado que realize $\mathrm{EF}$ de intensidade regular $\mathrm{e}$ moderada, com atividades prescritas em todas as fases da gestação e principalmente considerando as possíveis patologias gestacionais, como a SHG. Vale ressaltar que a orientação, o acompanhamento e as intervenções de um profissional de saúde habilitado e qualificado são de suma importância. A prática do EF deve ser realizada durante as diversas fases da vida, sendo assim uma das funções ideais para uma boa manutenção da forma física, inclusive durante a gestação. No entanto, quando se trata desse público alvo, todo EF deve ser feito de forma personalizada, levando em consideração cada gravidez, de modo que a gestante não deve ultrapassar seus limites.

Re-significação da TES para a adesão do exercício físico com vista à prevenção e/ao controle do risco da $\mathrm{SHG}$

Observamos nos relatos das gestantes, elementos importantes: adesão ao EF, benefícios percebidos com a prática do EF, agente multiplicador, e continuidade e planos para o futuro.

\section{Adesão ao Exercício Físico}

[...] olha, eu todo dia arranjo uma horinha enquanto meu bebê está dormindo, deixo no bebê-conforto com a minha vizinha ou com meu marido quando ele está em casa, calço meu tênis e desço para caminhar. Já faz quase um mês que eu estou caminhando todos os dias de segunda a sexta. Fico de 40 minutos a lhora e estou suando bastante, chego em casa cansada [...] aí depois tomo um banho e me sinto outra [...] fico muito mais disposta para cuidar do meu filho e fazer as minhas atividades de dona de casa (G3, 27 anos).

Ferreira et $\mathrm{al}^{(33)}$, em estudo com sessenta gestantes que frequentavam regularmente o $\mathrm{PN}$, questionaram sobre a participação delas em algum programa de EF, e registraram que quatorze gestantes informaram que às vezes participavam de programa de $\mathrm{EF}$, trinta gestantes não frequentavam, e dezesseis gestantes declararam que eram assíduas.

Observamos que a prática do EF durante o período gestacional ainda não está muito difundida na sociedade, visto que, normalmente, as gestantes não praticavam nenhum tipo de modalidade esportiva durante esta fase. São raras as gestantes que relatavam a participação regular em um programa de EF, o que mostra a falta de conhecimento sobre os benefícios desta prática durante o período gravídico, ou caso conheçam, há a ausência de engajamento na mesma.

\section{Benefícios do EF}

[...] está vendo como eu já estou mais magra? Eu já estou sentindo de novo os benefícios do EF no meu corpo, já comecei a diminuir de peso, estou sentindo menos fome porque eu passava o dia em casa com o neném aí fica só comendo, mas depois que eu voltei a caminhar me sinto muito melhor, com menos fome, mais ativa, menos inchada [...] passo o dia esperando dar 16 h para eu ir caminhar porque realmente estou me sentindo bem, sei que estou perdendo peso, diminuindo minha pressão $e$ fazendo bem para minha saúde (G3, 27 anos).

Um estilo de vida saudável que inclua regularmente o EF, é um elemento de extrema relevância na prevenção e no controle de determinadas doenças crônicas, como as cardiovasculares, dentre elas a mais importante é a HAS, a obesidade e a dislipidemia, e na redução da morbidade e mortalidade por muitas outras patologias ${ }^{(31)}$. Visualizamos entre as gestantes, que houve uma redução gradual da PA Média (PAM) após a realização do programa de caminhada. Mediante a comparação da PAM, antes e após a prática da caminhada, notamos que as gestantes, com exceção de uma, obtiveram menor média da PAM. No último encontro, as gestantes apresentavam valores PAM abaixo dos quais 
iniciaram o programa de caminhada. Registramos uma oscilação da PAM nas gestantes em cada encontro, antes da prática da caminhada e após trinta minutos de repouso da caminhada. Notamos que ao longo dos encontros, o valor da PAM de todas as gestantes foi menor em relação aos que elas iniciaram o programa de caminhada.

Nascimento et al $^{(32)}$ afirmam que a indicação do EF na gravidez, inclusive para as gestantes obesas e hipertensas, já é considerada uma prática segura, e deve ser conhecida e orientada por toda equipe de profissionais da saúde. No entanto, novos estudos devem ser realizados com objetivo de preencher as arestas sobre recomendações de intensidade, frequência e duração do EF, a partir de métodos diretos de avaliação do bem-estar fetal.

De acordo com Domingues e $\operatorname{Barros}^{(37)}$ a caminhada controlada é o programa de EF mais comum entre as gestantes, e é efetiva no controle das $\mathrm{SHG}$, e na prevenção e controle do excesso de ganho de peso na gestação, que também corroboram para o surgimento destas.

Alves e Forjaz ${ }^{4)}$ afirmam que um treinamento aeróbico com indivíduos hipertensos reduz a PA sistólica/diastólica em média de 6,9/4,9 $\mathrm{mmHg}$, porém a grandeza desta redução é influenciada pelas características do treinamento. De modo geral, observa-se ainda escassez de dados sobre este assunto, mas os existentes sugerem que o maior efeito hipotensor é promovido por EF de intensidade leve e com volume semanal elevado, o que pode ser alcançado com sessões de maior duração e/ou maior frequência semanal. Assim, para pessoas hipertensas, o treinamento aeróbico deve ser composto por sessões de pelo menos trinta minutos, realizadas com uma frequência semanal mínima de três vezes e com intensidade leve (40,0 a 60,0\% do VO2 max).

\section{Agente Multiplicador}

[...] hoje mesmo eu convenci minha cunhada e nós combinamos de caminhar hoje à tarde. Ela também teve bebê e eu disse que era bom para ela, para saúde dela e para evitar a pressão alta, né? [...] (G5, 16 anos).

Para Prochaska, Norcross e Di Clemente ${ }^{(27)}$, um dos aspectos importantes a ser verificado no transformado é que este se torna um agente multiplicador, ou seja, o mesmo se sente tão beneficiado com os alcances adquiridos e empolgado com o novo estilo de vida que faz questão de compartilhar com familiares e amigos para que venham a sentir também os benefícios por ele alcançados. Santos, Caetano e Moreira ${ }^{(38)}$ constataram que os pais de crianças com propensão à HAS revelaram-se agentes multiplicadores do saber sobre a prevenção deste agravo na família. Contudo, a missão de educador não tem prazo delimitado, e, a exemplo da aprendizagem, é algo inesgotável. No cotidiano, somos sempre agentes de mudança e multiplicadores. Assim, com a aplicação de mudanças no cotidiano conseguiram alcançar os objetivos desejados, assim como as gestantes desse estudo que passaram a convidar parentes e amigos a praticarem EF. A educação em saúde (ES) encontrase embasada na concepção de promoção da saúde, que aborda os métodos que contemplam a cooperação de toda a população no âmbito de sua vida cotidiana e não somente daquelas pessoas sob o risco de adoecimento. Essa questão está ancorada em um conceito de saúde, sendo apreciado como positivo e à procura do bem-estar, agregando aspectos físicos, mentais, ambientais, pessoal e social ${ }^{(39,40)}$.

As ações de ES encontram-se relacionadas ao exercício da cidadania na busca por melhores condições de vida e de saúde da população, de modo a despertar comportamentos, valores e atitudes entre os indivíduos, como forma de estimular o processo de reflexão sobre como buscar informações sobre comportamentos, valores, crenças e atitudes frente aos fatores de risco para $\mathrm{SHG}$, com vista ao planejamento de programa educativo para a população em estudo $^{(38,40)}$

Continuidade da prática do exercício físico e Planos para o futuro

[...] eu quero voltar a fazer caminhada, academia e ficar correndo para vida toda para ficar sempre com saúde (G7, 16 anos).

Prochaska, Norcross e Di Clemente ${ }^{(27)}$ asseguram que as pessoas que conseguem finalizar o processo de transformação nunca mais abandonam o seu novo hábito e querem sempre continuar sentindo os benefícios alcançados. Esse processo passa a ser extremamente fácil e natural.

\section{CONSIDERAÇÕES FINAIS}

A aplicação da TES conduziu as gestantes ao “estágio de manutenção", significando que elas aderiram ao EF, e como conduta promotora de saúde. No entanto, ainda é necessário incentivo para mantê-las neste estágio, ou elevá-las à terminação, mediante a incorporação desta conduta no seu estilo de vida. Após a gestação, três gestantes continuaram efetivamente realizando o programa de caminhada, o que nos leva a notar que alcançaram o "estágio de terminação", enquanto duas gestantes retrocederam quanto ao nível de mudança anteriormente adquirido, mostrando, assim, dificuldade na adesão à prática do EF.

Diante desses fatos, é de extrema importância que ações de ES permeiem a vida dessas mulheres, com o intuito de garantir uma gravidez saudável, educando-as para adquirirem conhecimento quanto à mudança dos comportamentos de risco para estilos preventivos e de controle de agravos na gravidez, principalmente a SHG. 
É importante ressaltar a necessidade dessas mulheres se tornarem agentes multiplicadoras do conhecimento, buscando conscientizar, cada vez mais, pessoas a aderirem à prática de estilo de vida saudável e incorporarem o EF no seu cotidiano, como forma de garantir qualidade de vida e bem-estar.

Os resultados deste estudo possibilitarão uma reflexão à EqSF sobre a articulação com o Núcleo de Apoio a Saúde da Família (NASF) sobre a incorporação desta TES com vista à prevenção e/ou controle do risco da SHG, com a manutenção da PA controlada. Sendo assim, uma tecnologia voltada para o combate ao sedentarismo através da adesão da gestante ao EF poderá contribuir para a promoção de sua saúde.

O NASF é composto por vários profissionais que dão apoio matricial a EqS, dentre estes o Educador físico e o Fisioterapeuta que podem se envolverem nas ações educativas com mulheres grávidas. Nesse contexto, é de extrema importância à implementação de práticas de EF com acompanhamento destes profissionais que integram o NASF, direcionadas às gestantes, possibilitando, assim, a mudança de comportamentos de risco para prevenção e controle de agravos na gravidez, principalmente a SHG, através de mudanças significativas no estilo de vida, conduzindo-as a uma gravidez saudável, regada de bem-estar e vivida em toda sua plenitude. Vale ressaltar a necessidade de haver um acompanhamento mais prolongado com essas mulheres com o intuito de promover mudanças de comportamento duradouras, não apenas motivadas de forma pontual e sem continuidade. Esse tipo de prática deve ser uma ação rotineira da equipe que integra o NASF.

Ressalta-se que as condutas do SS brasileiro, que embora ainda apresente inúmeras dificuldades, existe a preocupação com a saúde da gestante, disponibilizando um serviço eficiente de PN através da Estratégia de Saúde da Família (ESF). A adesão das mulheres ao PN está relacionada com a qualidade de assistência prestada pelo serviço e pela Equipe de Saúde. A atenção dada ao PN contribui significativamente na redução das taxas de morbimortalidade materna, promovendo, dessa forma, uma maternidade segura. Esse cuidado é um desafio a ser vencido e está diretamente ligada ao bom desempenho por parte da Equipe de Saúde que assiste as gestantes, prestando assim um serviço de excelência. Logo, o aprimoramento técnico e científico da Equipe de Saúde muito pode contribuir no acompanhamento dessas gestantes e, consequentemente, alcançar o equilíbrio e o bem-estar, tanto materno quanto fetal, nos procedimentos diagnósticos e terapêuticos.

Esse estudo possui limitações, como a escassez de literatura sobre a temática do EF na gestação, e por ser um tema que ainda gera controvérsias no meio médico e leigo acerca dos possíveis riscos que o EF pode trazer à saúde da gestante e do concepto. Ressaltamos que esse estudo não se esgota, principalmente, nesse contexto em que se torna necessária a realização de novos estudos para elucidar os benefícios, indicações e contraindicações do EF durante a gestação. Como também, é importante de se continuar pesquisando os diversos contextos acerca da SHG, que podem ser imprescindíveis para o fomento de propostas de intervenção com mulheres que podem ser acometidas dessa intercorrência obstétrica.

\section{REFERÊNCIAS}

1. Alves SV. Maternal Mortality in Pernambuco, Brazil: What Has Changed in Ten Years? Reproductive Health Matters. 2010,45(30):336-55.

2. Sociedade Brasileira de Cardiologia. Diretrizes Brasileiras de Hipertensão Arterial. Arquiv. Bras. Cardiol. 2016;107(Supl 3):1-83.

3. Ministério da Saúde (BR). Secretaria de Atenção à Saúde. Departamento de Ações Programáticas Estratégicas. Gestação de alto risco: manual técnico / Ministério da Saúde, Secretaria de Atenção à Saúde, Departamento de Ações Programáticas Estratégicas. 5. ed. Brasília: MS; 2010a. 302 p.

4. Alves, LL, Forjaz C. Influência da Intensidade e do Volume do Treinamento Aeróbico na Redução da Pressão Arterial de Hipertensos. Revista Bras. Ci e Mov. 2009;15(3):115-22.

5. Kale PL, Costa AJL. Maternal Deaths in the City of Rio de Janeiro, Brazil, 2000-2003. Health Popul Nutr. 2009;27(6):794-801.

6. Mota SMS, Gama SGN, Theme MM. Mortalidade materna no Município de Belém, Estado do Pará, em 2008: Uma avaliação do Sistema de Informações sobre Mortalidade. Epidemiol. Serv. Saúde, Brasília. 2009;17(1):33-42.

7. Sass $\mathrm{N}$ et al. Maternal mortality in Brasil and proportion to hypertensive disorders: a trend of stagnation. Pregnancy Hipertens. 2015; 5(1):78-92.

8. Brasil. Ministério da Saúde. Secretaria de Atenção à saúde. Departamento de Ações Pragmáticas Estratégicas. Gestação de alto risco. Manual técnico. $5^{\mathrm{a}}$ ed. Brasília: MS; 2012.

9. Ministério da Saúde (BR). Departamento de Informática do Sistema Único de Saúde (DATASUS). Brasília: MS; 2010b.

10. Ministério de Saúde (BR). Sistema de Informação sobre Mortalidade (SIM). Brasília: MS; 2010c. 
11. Ministério da Saúde (BR). Secretaria de Atenção à Saúde. Departamento de Atenção Básica. Política Nacional de Atenção Básica. 4. ed. Brasília: Série Pacto pela Saúde; 2006. v.4.

12. Ximenes Neto FRG et al. Qualidade da atenção ao prénatal na Estratégia Saúde da Família em Sobral, Ceará. Rev. Bras. Enferm. 2008;61(5):595-602.

13. Reis AE, Patricio AM. Aplicação das ações preconizadas pelo Ministério da Saúde para o parto humanizado em um hospital de Santa Catarina. Ciências da Saúde Coletiva. 2010;10 (Supl): 221-30.

14. Andreucci CB, Cecatti JG. Desempenho de indicadores de processo do Programa de Humanização do Pré-natal e Nascimento no Brasil: uma revisão sistemática. Cad. Saúde Pública. 2011;27(6):1053-64.

15. Serruya SJ, Cecatti JG, LagoTG. O Programa de Humanização no Pré-natal e Nascimento do Ministério da Saúde no Brasil: resultados iniciais. Cad. Saúde Pública. 2004;20(5):1281-89.

16. Almeida CAL, Tanaka OY. Perspectiva das mulheres na avaliação do Programa de Humanização do Pré-Natal e Nascimento. Rev Saúde Pública. 2009;43(1):98-104.

17. Matos DS, Rodrigues MS, Rodrigues TS. Atuação do enfermeiro na assistência ao pré-natal de baixo risco na estratégia saúde da família em um município de Minas Gerais. Rev Enferm. 2013; 16(1):18-33.

18. Ribeiro JF et al. Contribuição do pré-natal para o parto normal na concepção do enfermeiro da estratégia saúde da família. R. Interd. 2016;9(1):161-170.

19. Sayem AM, Nury ATMS. Factors associated with teenage marital pregnancy among Bangladeshi women. Reproductive Health. 2011;8(16):1-12.

20. Morais FM et al. Uma revisão do perfil clínicoepidemiológico e das repercussões perinatais em portadoras de síndrome hipertensiva gestacional. Revista EIXO. 2013; 21(1): 69-82.

21. Peracoli JC, Parpinelli MA. Síndromes hipertensivas da gestação: identificação de casos graves. Rev. Bras. Ginecol. Obstet. 2011, 47 (10): 123-38.

22. Sorensen TK et al. Recreational physical activity during pregnancy and risk of preeclampsia. Revista Hypertension. 2010; 81(3): 1273-80.

23. American College of Obstetricians and Gynecologists (ACOG). Committee on Obstetric. Exercise during pregnancy and postpartum period. Pratice $n^{\circ} 267$. Am Col Gynecol. 2012, 99 (4): 171-73.
24. Oliveira EFV, Gama SGN, Silva CMFP. Gravidez na adolescência e outros fatores de risco para mortalidade fetal e infantil no Município do Rio de Janeiro, Brasil. Revista Cad. Saúde Pública. 2010;26(3):384-97.

25. Lima FR, Oliveira N. Gravidez e Exercício. Rev Bras Reumatol. 2011;45(3):188-90.

26. Rudra $\mathrm{CB}$ et al. Perceived exertion during prepregnancy activity and preeclampsia risk. Med Sci Sports Exerc. 2010;37(11):1836-41.

27. Prochascka JO, Norcross JC, DI Clemente CC. Changing for Good. A revolutionary six-stage program for overcoming bad habits and moving tour life positively forward. New York: Avon Books; 1994.

28. Jalowitzki M. Vivências para dinâmicas de grupos - a metamorfose do ser em 360 graus. São Paulo: Masdras; 2007.

29. Bardin L. Análise de conteúdo. São Paulo: Edições 70; 1994.

30. Ministério da Saúde (BR). Resolução No 466 de 12 de dezembro de 2012. Regulamentação da Pesquisa envolvendo seres humanos. Brasília: CONEP/CNS/ MS; 2012.

31. Segato L et al. Ocorrência e controle do estresse em gestante e sedentárias e fisicamente ativas. Revista da Educação Física/UEM Maringá. 2010;22(1):171-89.

32. Nascimento SL et al. Exercício físico no ganho de peso e resultados perinatais em gestantes com sobrepeso e obesidade: uma revisão sistemática de ensaios clínicos. Cad. Saúde Pública. 2011, 27(3):407-16.

33. Ferreira IP et al. Gestantes: conhecendo os benefícios da prática de exercícios físicos em uma unidade de saúde da família. Revista Anais da Semana Educa. 2010;1(10):20-25.

34. Gus M, Fuchs FD, Moreira LB. Hipertensão associada a gestação e risco cardiovascular. Hipertensão 2010; 14(1): 78-89.

35. Matsudo VKR, Matsudo SMM. Atividade Física e Esportiva na Gravidez. In: TEDESCO JJ. A Grávida. São Paulo: Atheneu; 2010. p.156-78.

36. Batista DC et al. Atividade física e gestação: saúde da gestante não atleta e crescimento fetal. Revista Bras. Saúde Mater. Infantil. 2011;18(2):351-68.

37. Domingues MR, Barros AJD. Leisure-time physical activity during pregnancy in the 2004 Pelotas Birth Cohort Study. Rev Saúde Pública. 2010;41(4):173-80. 
38. Santos ZMSA, Caetano JA, Moreira FGA. Atuação dos pais na prevenção da hipertensão arterial: uma tecnologia educativa em saúde. Ciênc. Saúde Coletiva. 2011;16(11):4385-94.

39. Moura ERF, Souza RA. Educação em saúde reprodutiva: proposta ou realidade do Programa Saúde da Família? Revista Cad Saúde Pública. 2012;28(6):1809-11.

40. Araújo FM. Ações de educação em saúde no planejamento familiar nas unidades de saúde da família no município de Campina Grande PB (Monografia de
Especialização). Universidade Estadual da Paraíba. Campina Grande; 2011.

\section{Endereço para correspondência:}

Zélia Maria de Sousa Araújo Santos Universidade de Fortaleza - UNIFOR

Programa de Pós-Graduação em Saúde Coletiva - PPGSC Av. Washington Soares, 1321 bloco S Bairro: Edson Queiroz CEP 60811-905 - Fortaleza - CE - Brasil Email: zeliasantos@unifor.br 